\title{
Spatial context affects the Poggendorff illusion
}

\author{
MICHAEL J. SPIVEY-KNOWLTON \\ University of Rochester, Rochester, New York \\ and \\ BRUCE BRIDGEMAN \\ University of California, Santa Cruz, California
}

\begin{abstract}
The Poggendorff illusion has often been explained as purely an interaction between the parallels and the transversals. The present study demonstrates that additional spatial context exerts an influence on this illusion. In Experiment 1, we examined the effects of a surrounding tilted frame (complete and degraded versions) on collinearity adjustments in the upright and rotated Poggendorff figures. The frame's orientation was always oblique. Relative to the no-frame condition, frames decreased error in collinearity adjustments in the upright Poggendorff figure, and increased error in the rotated Poggendorff figure. In Experiment 2, a circumscribing circle did not cause an orientation-inhibition effect (Ebenholtz \& Utrie, 1982, 1983), suggesting that the effect of the frame on the Poggendorff illusion may not be closely related to the rod-and-frame effect. In Experiment 3 , orientation of a central texture modulated the magnitude of the illusion. The results do not serve to explain the mechanisms behind the Poggendorff illusion, but they do demonstrate the importance of visual reference frames in understanding perceived misalignment.
\end{abstract}

Most research on the Poggendorff illusion has focused on explaining the effect within the scope of the lines in the Poggendorff figure itself (Figure 1A). The results have suggested that the effect is attributable to an increase in perceived size of the smaller angle and/or a decrease in perceived size of the larger angle (Helmholtz 1910/1962; Hotopf \& Ollerearnshaw, 1972; Imai, 1973), and/or to perceived rotation of the transversals toward perpendicular with respect to the parallels (Hotopf, Ollerearnshaw, \& Brown, 1974; Krantz \& Weintraub, 1973; Weintraub \& Krantz, 1971; Weintraub, Krantz, \& Olson, 1980). However, the illusion still exists when presented with only dots instead of transversals (Coren, 1970; Day \& Dickinson, 1976; Day \& Kasperczyck, 1985; Predebon, 1983; Tong \& Weintraub, 1974; Wenderoth, Beh, \& White, 1978; Wenderoth $\&$ Wade, 1981), thus ruling out perceived angle size and rotation as comprehensive explanations.

Others have suggested that the illusion is due to perceived narrowing of the gap between the two parallels (Day \& Dickinson, 1976; Greist-Bousquet \& Schiffman, 1981; Quina \& Pollack, 1973; Zanuttini, 1976). While this explanation has survived many of the variants of the Poggendorff illusion, it doesn't naturally predict the reduction in the illusion when the figure is rotated (Fig-

This research was supported in part by AFOSR Grant 90-0095 to B.B. and by a Sproull Graduate Fellowship from the University of Rochester and a Research Training Grant Award from the National Research Council to M.S.-K. The authors wish to thank Mark Goettsche for software and apparatus design, Caryn Nardello for assistance in data collection, and Mary Hayhoe, Dorm Massaro, Alexander Pressey, Dan Weintraub, and an anonymous reviewer for helpful comments on earlier versions of the manuscript. Correspondence should be addressed to $\mathbf{M}$. SpiveyKnowlton, Center for Visual Science, Meliora Hall, University of Rochester, Rochester, NY 14627 (e-mail: spivey@cvs. rochester.edu). ures $1 \mathrm{~B}-1 \mathrm{C}$ ), or the persistence of the illusion (albeit weakened) when the parallels are removed (Figure 1D).

Pressey (1971) has suggested the integrative field theory, in which such orientation illusions are explained in terms of a weighted average of the inducing lines "pulling" the perceived point of intersection away from the veridical intersection. Similarly, a "cognitive mistracking" explanation claims that as the viewer mentally extends one of the transversals in the Poggendorff illusion, his/her trajectory deviates toward perpendicular with respect to the inducing lines (Tong \& Weintraub, 1974; Weintraub \& Krantz, 1971). While somewhat vague and heuristic, this account seems compatible with most of the psychophysical data in the literature. However, it also offers no explanation for the "oblique-line illusion" (Day, 1973; Goldstein \& Weintraub, 1972; Wundt, 1893), in which two oblique and collinear segments alone appear misaligned in the same direction as in the Poggendorff illusion, but to a lesser degree (Figure 1D).

In more recent work, Hotopf (Findlay \& Hotopf, 1985; Hotopf, 1981) has proposed a kind of mistracking explanation that is similar to Weintraub's, in which the inducing line's "pull" on the transversal's imaginary extension is described as a "center of gravity" effect. The crucial difference between Weintraub's "cognitive mistracking" and Hotopf's "attraction misalignment" is that Hotopf's version is directly related to eye movements, whereas Weintraub's explanation specifically avoids the postulation of a direct relationship between eye movements and the tracking strategy.

Without a straightforward (and non-strategy-specific) link to neurophysiology or some theoretical context, many of these explanations appear to be little more than redescriptions of the phenomena. To our knowledge, the only gen- 


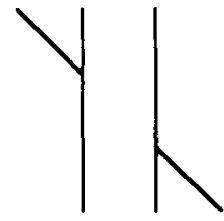

A

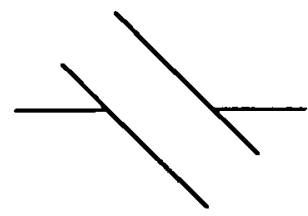

C
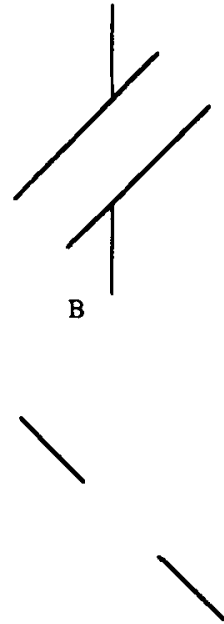

$\mathrm{D}$
Figure 1. The common version of the Poggendorff illusion (A). Both rotated Poggendorff figures ( $B$ and $C$ ) produce weaker illusory effects. Even the Poggendorff without parallels (D) still has a mild illusion of misalignment.

eral visual process that has been invoked to explain the full range of Poggendorff illusion phenomena (not just small angular separations, as in angular repulsion theories) is depth perception (Gillam, 1971, 1980; Green \& Hoyle, 1964; Gregory, 1966). As with the theories of perceived rotation and angle size, similar results with dots instead of transversals suggest that this explanation is inadequate.

However, if any combination of these theories is to be a complete explanation of the Poggendorff illusion, it must address and account for any possible reference-frame effects or influences from additional contours. For example, a simple tilted frame greatly affects an observer's perception of true vertical (Asch \& Witkin, 1948; Beh, Wenderoth, \& Purcell, 1971; Ebenholtz, 1977, 1985a, 1985b, 1985c; Ebenholtz \& Utrie, 1982, 1983; Wenderoth, 1974; Witkin \& Asch, 1948). It is conceivable that a similar frame may affect an observer's perception of collinearity in the Poggendorff illusion. In fact, Pressey (1967) found a significant correlation between subjects' performance on the Poggendorff illusion adjustment task and on the rod-and-frame task.

In the classic rod-and-frame experiment, subjects are placed in a dark room with a luminous rod surrounded by a luminous frame (tilted usually around $22^{\circ}$ ) and instructed to manually rotate the rod to true (gravity-based) vertical. Generally, subjects place the rod at $8^{\circ}-9^{\circ}$ toward the tilt of the frame. This result has been described as an "automatic influence of the peripheral representation of the frame on the egocentric orientation-coordinate system," biasing the perception of true vertical toward the frame's own vertical and horizontal (Ebenholtz, 1985b, "p. 305). Moreover, adding a circular contour that surrounds the frame tends to inhibit the effect of the frame to about $30 \%$ of normal magnitude, but placing the same circle inside a frame produces no effect (Ebenholtz \& Utrie, 1982, 1983). This has been called the "orientationinhibition effect," and it is proposed that it is caused by a greater orientation influence from peripheral (or outermost) reference frames than from central ones.

Indirect tilt illusions (attraction of orientations) are also affected by frame contours (Wenderoth \& Johnstone, 1988; Wenderoth \& van der Zwan, 1989). When a central vertical grating is surrounded by an annulus grating tilted between $50^{\circ}$ and $85^{\circ}$ clockwise, the central grating will appear tilted slightly toward clockwise. Peak indirect tilt illusions $\left(.5^{\circ}-1.5^{\circ}\right)$ occur with $70^{\circ}-80^{\circ}$ angular separation. However, when an upright frame is introduced around the display, the illusion vanishes. Curiously, the same is not true for direct tilt illusions (repulsion of orientations at smaller angular separations) (Wenderoth \& Johnstone, 1988; Wenderoth \& van der Zwan, 1989). It has been proposed (Wenderoth, van der Zwan, \& Johnstone, 1989), and supported by a computational model (Spivey-Knowlton, 1992), that direct tilt illusions are caused by lateral inhibition between orientation channels and that indirect tilt illusions are caused by lateral inhibition from a "virtual main line axis" that is orthogonal to the inducing stimulus.

We propose that, if the Poggendorff illusion is susceptible to an influence from a reference frame (or an internal grating), competing theories of the Poggendorff illusion must be updated to incorporate a broader range of determinants. A simple demonstration of this contextual dependence can be observed by looking at a rotated Poggendorff figure (Figure 1B or 1C) and then tilting one's head to the orientation of the parallels. The illusory effect is rather small when the figure is viewed with the head upright. However, tilting the head aligns the head-centered reference frame with the parallels of the figure (as is the case when one views the upright Poggendorff with the head upright), and the illusion increases to near-normal magnitude.

To test systematically the effect of a surrounding visual reference frame on the Poggendorff illusion, in Experiment 1 the subjects were required to make collinearity adjustments on upright and rotated Poggendorff figures in full-frame, various degraded-frame, and no-frame blocked conditions. To explore the compatibility of this frame effect with that of the rod-and-frame paradigm, via Ebenholtz's orientation-inhibition effect, in Experiment 2 three frame conditions were cross-indexed by a surrounding circle condition on both upright and rotated figures. In Experiment 3, a grating was introduced in the region between the parallels of the Poggendorff figure, rather than a frame surrounding it. This experiment tested the upright and rotated figures with several vertical segments, horizontal segments, or both, in between the parallels.

\section{EXPERIMENT 1}

Clearly, a reference-frame proposal cannot on its own predict the directionality of perceived misalignment. It 
merely predicts that, as the orientation of the reference frame gets farther from that of the alignment task, accuracy (indicating veridical alignment perception) will decline. The directionality of perceived misalignment must be predicted by more specific mechanisms, such as those outlined in the introduction.

\section{Method}

Subjects. Twelve undergraduates participated in this experiment to fulfill a course requirement. All had normal or corrected vision and were naive with regard to the experimental manipulations.

Apparatus. The stimuli were presented on a Hewlett-Packard $1310 \mathrm{~A}$ vector screen and automatic data collection was controlled by an IBM 386 Model 70 computer. Placed over the vector screen was a black cloth with a rectangular aperture approximately the same size as the screen itself but with rounded corners. This was to prevent the shape of the screen from providing a salient frame of reference. A chin and forehead rest provided a stabilized viewing distance of $57 \mathrm{~cm}$, so that $1 \mathrm{~cm}$ on the screen subtended about $1^{\circ}$ of visual angle.

Even with all lights off in the experimentation room, dark adaptation allowed some of the walls to be seen eventually, thus providing a more peripheral reference frame than that of the stimulus frame. To exclude this reference frame, a black cloth was draped over the forehead rest with a circular viewing aperture for the right eye only; this allowed a view of the vector screen but little or none
A

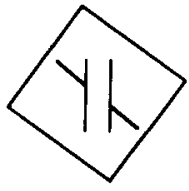

B

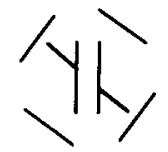

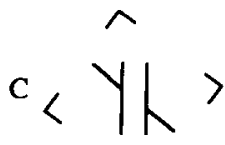<smiles>[13CH3]</smiles>

D<smiles>CC(C)C</smiles>

$\mathrm{E}$

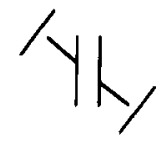

$\mathbf{F}$

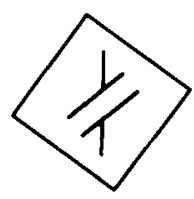

G

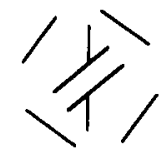

$<\overbrace{\frac{1}{\gamma}}\rangle$

I

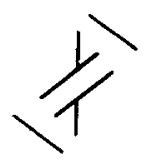

J

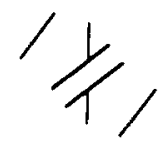

Figure 2. Examples of stimuli for Experiment 1. (A) Full frame. (B) Segment frame. (C) Vertices frame. (D) Half-left frame. (E) Half-right frame. The complementary rotated versions follow. of the surrounding area. The brightness of the vector screen was adjusted to the subject's preference before experimentation began.

With the stimulus frame tilted at $45^{\circ}$, the subjects would have been able to align the vertical transversals of the rotated Poggendorff figure with the bottom corner of the stimulus frame. To prevent this alternate strategy, stimulus frames were rotated at $55^{\circ}$ counter-clockwise, and the transversals of the Poggendorff figure were placed at $55^{\circ}$ angular separation from the parallels. The six frame conditions included no-frame, full-frame, segment-frame, vertices-frame, half-left-frame, and half-right-frame conditions. See Figure 2 for the frame and its variants.

The two parallels of the Poggendorff figure subtended $10^{\circ} \times$ $2.5^{\circ}$ of visual angle. The transversals were $3.5^{\circ}$ of visual angle in length, and the full frame subtended $17.2^{\circ}$ of visual angle. For both the segment frame and the vertices frame, exactly $80 \%$ of the frame was deleted. The half-frames were constructed from the segments of the segment frame (see Figure 2).

Manual response was made by an analog velocity knob that moved the right-hand transversal either up or down. The subject pressed a "set" button to complete each trial, after which the stimulus was erased and any screen persistence was blotted out by a mask before the next trial began.

Procedure. Twenty trials for each of the six frame conditions were presented in blocked sequence. Ten upright and 10 rotated figure trials were randomized within each block. In each trial block, the mobile transversal started in a position randomly varying between .5 and $1 \mathrm{~cm}$ from the upper end or the lower end (for 10 randomized trials each) of the right-hand parallel. The sequence of the trial blocks was randomized between subjects with (approximately) 3-min breaks in between.

Before data collection began, the subjects were required to perform two demonstration trials to ensure that they understood the alignment task and knew how to use the velocity knob. The subjects were instructed to stop the mobile transversal at the perceived collinear position before pressing the "set" button, and to spend no more than $20 \mathrm{sec}$ or so on each trial. The experiment lasted approximately $1 \mathrm{~h}$.

\section{Results}

A repeated measures analysis of variance for the error for all 12 subjects produced the predicted interaction of frame $\times$ rotation (Figure 3 ). Generally, the frame and its variants facilitated accuracy in the upright figure and inhibited accuracy in the rotated figure $[F(5,55)=5.36$, $p<.001]$. Fisher's least significant difference tests (see Carmer \& Swanson, 1973) were applied to the cells of this significant interaction to determine which frame components were instrumental in producing this effect. The upright no-frame condition produced a significantly greater magnitude of illusion than did the rotated no-frame condition $(p<.01)$. The upright no-frame condition also significantly exceeded the upright half-right-frame condition $(p=.02)$ and the upright vertices-frame condition $(p<.05)$. The upright full-frame, segment-frame, and half-left-frame conditions did not, however, differ significantly from the upright no-frame condition. The rotated no-frame condition produced a significantly lesser magnitude of illusion than did the rotated full-frame condition $(p<.01)$, the upright full-frame condition $(p<$ $.02)$, the rotated segment-frame condition $(p<.02)$, the upright segment-frame condition $(p<.05)$, the rotated half-left-frame condition $(p<.001)$, and the rotated halfright-frame condition $(p<.02)$. 


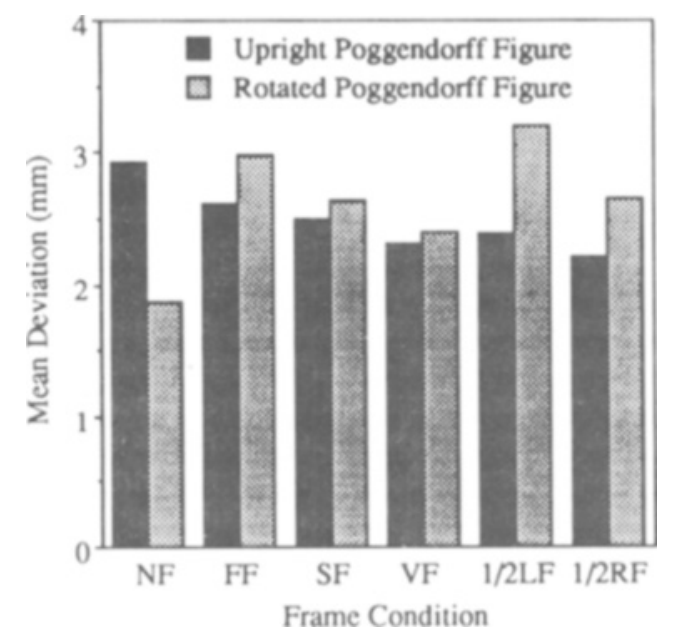

Figure 3. The effect of figure rotation as a function of frame condition (NF, no frame; FF, full frame; SF, segment frame; VF, vertices frame; $1 / 2 \mathrm{LF}$, half-left frame; $1 / 2 R F$, half-right frame). In the no-frame condition, the common decrease in illusory effect for the rotated figure (in comparison with the upright figure) is exhibited. This relationship disappears, however, when the frame or one of its variants is present.

Also observed was a very robust main effect of starting position such that when the mobile transversal started in the lower position subjects made smaller errors $(1.65 \mathrm{~mm})$ than when it started in the upper position $[3.46 \mathrm{~mm} ; F(1,11)=$ $40.02, p<.001]$. A significant interaction of frame $\times$ starting position was also observed in the analysis. The difference between the errors for the two starting positions was smaller in the full-frame and half-right-frame conditions (starting position difference $=$ full frame, $1.37 \mathrm{~mm}$; halfright-frame, $1.22 \mathrm{~mm}$ ) than in the other frame conditions [starting position difference $=$ no frame, $2.01 \mathrm{~mm}$; segment frame, $2.16 \mathrm{~mm}$; vertices frame, $2.18 \mathrm{~mm}$; halfleft-frame, $1.94 \mathrm{~mm} ; F(5,55)=2.86, p<.05$ ].

Another significant effect was observed in the rotation $\times$ starting position interaction. A greater starting position effect was found in the upright figure (upper starting position, $3.72 \mathrm{~mm}$; lower, $1.26 \mathrm{~mm}$ ) than in the rotated figure [upper starting position, $3.21 \mathrm{~mm}$; lower, $2.04 \mathrm{~mm}$; $F(1,11)=7.5, p<.05]$.

\section{Discussion}

The effect of the frame and its variants supports our hypothesis that one component of the Poggendorff illusion is the role of the reference frame. The fact that certain variants of the frame decreased error for the upright figure and increased error for the rotated figure fits well into our proposal that a visual reference frame provides coordinate axes upon which similarly aligned line segments (such as the transversals of the upright Poggendorff) may be more accurately indexed for collinearity. Moreover, this tilted visual reference frame is likely to interfere with the head-, body-, and gravity-centered refer- ence frames that normally facilitate collinearity matching in the rotated Poggendorff.

Reference frames do not provide a complete explanation for the Poggendorff illusion, for two reasons. First, as mentioned before, they are unable to predict the direction of the perceived noncollinearity. Second, the presence of the frame and its variants only mildly decreased the illusory effect for the upright figure. Thus, there are determinants of the Poggendorff illusion that are not counteracted by transversally aligned reference frames. Indeed, there are likely to be several factors that combine simultaneously to produce the illusion (Day \& Kasperczyck, 1985; Weintraub et al., 1980), some of which are affected by reference frames and some of which are not.

Although the effects of starting position were not expected, they may be explained in terms of a distinction between the perception of real and illusory transversal alignment. When the mobile transversal started above the true collinear position and was moved downward toward it, the illusory alignment was reached before true alignment was reached. Thus, the subject often stopped the transversal well before reaching the true collinear position. Starting from below the true collinear position, however, the mobile transversal reached the true alignment position before reaching the illusory alignment position.

\section{EXPERIMENT 2}

The rod-and-frame effect has been shown to decrease when the frame is surrounded by a circular contour (Ebenholtz \& Utrie, 1982, 1983). Indirect tilt illusions, but not direct tilt illusions, are also decreased by the presence of a surrounding circle (Wenderoth \& Johnstone, 1988). To test the compatibility of the reference-frame effect on collinearity adjustments observed in Experiment 1 with the rod-and-frame effect and the indirect tilt illusion, in Experiment 2 we introduced a circumscribing circular contour to the full-, segment-, and vertices-frame conditions of Experiment 1.

If the circle reduced the effect of the frame (both its inhibitory and its facilitatory effect), it would support a unification of the rod-and-frame effect and indirect tilt illusion with the present frame effect. If, however, an orientationinhibition effect was not obtained through the presence of the circle, it would suggest that two distinct mechanisms mediate the rod-and-frame effect and the indirect tilt illusion, on the one hand, and the frame's effect on the Poggendorff, on the other.

\section{Method}

Subjects. Twelve undergraduates participated in this experiment to fulfill a course requirement. All had normal or corrected vision and were naive with regard to the experimental manipulations.

Apparatus. The stimuli, design, and apparatus were identical to those of Experiment 1, except that only the full-frame, segmentframe, and vertices-frame conditions were presented, and a circular contour subtending $25.5^{\circ}$ of visual angle surrounded the frame on half of the trials. 
Procedure. Data collection proceeded in a fashion identical to that of Experiment 1 .

\section{Results}

A repeated measures analysis of variance computed for the errors revealed a main effect of starting position as before [upper starting position, $4.22 \mathrm{~mm}$; lower starting position, $3.02 \mathrm{~mm} ; F(1,11)=15.24, p<.005$ ], but no other significant result was obtained. The interaction of circle $\times$ rotation predicted by the orientation-inhibition effect did not approach significance $[F(1,11)=2.62, p>$ $.10]$. The mean difference in error between the upright and rotated figures in the circle absent condition was $.001 \mathrm{~mm}$, and the same mean difference within the circle present condition was only $.3 \mathrm{~mm}$ (in the direction opposite that predicted).

\section{Discussion}

Although the orientation-inhibition effect would predict that the presence of the circumscribing circle would inhibit the effect of the frame and thus produce the significant difference between rotation conditions found in the no-frame condition of Experiment 1, this did not occur. In fact, the circle present condition produced no significant difference between the upright Poggendorff figure and the rotated figure.

This suggests that the rod-and-frame effect and the indirect tilt illusion are governed by a mechanism distinct from one that causes this frame effect on the Poggendorff illusion. Although a more parsimonious theory would attribute all three effects to a unitary reference-frame explanation, it appears that we must separate these two effects. A possible explanation is that task differences are responsible for this dissociation. In the rod-and-frame task and in the indirect tilt illusion task, a subject is required to set a line or grating to true vertical or judge whether it is left or right of true vertical. Such a task may involve greater use of information regarding absolute spatial orientation than a task in which a judgment about verticality is not required, such as a collinearity adjustment task for oblique lines. Greater reliance on orientation information logically entails a greater susceptibility to degradation of that information source due to a circular contour that may "drown out" the frame's signal (Ebenholtz \& Utrie, 1982,1983 ).

It is, of course, possible that our stimuli simply weren't similar enough to Ebenholtz and Utrie's (1982, 1983) to produce their predicted effect. Our lack of an orientationinhibition effect may also be the result of statistical noise, rather than evidence against the applicability of the orientation-inhibition effect to this task. However, the very small absolute magnitude of our result argues against this interpretation.

\section{EXPERIMENT 3}

The purpose of this experiment was to further test the susceptibility of the Poggendorff illusion to spatial context. Because Wilson (1983) emphasized the importance of the region between the parallels, we manipulated the orientation of a texture placed in this region. Following Blix's (1902) version of the upright Poggendorff figure with $40+$ horizontal lines forming the region between the parallels, we constructed central textures of horizontal lines, vertical lines, and a grid texture of both. (These lines are, of course, tilted in the rotated Poggendorff figure, but for simplicity, we will continue to refer to these textures as horizontal and vertical.)

The horizontal and vertical textures each support onedimensional reference orientations, while the grid texture supports a full reference frame. Although a referenceframe theory alone cannot predict the directionality of the modulation of the illusion, it can predict the unsigned relationship between the three texture conditions. Logically, the effect of the horizontal texture on collinearity adjustments should be opposite that of the vertical texture, with the grid texture falling in between. More specifically, a description such as "cognitive mistracking" (Tong \& Weintraub, 1974; Weintraub \& Krantz, 1971) makes predictions for the direction of effect. It would logically predict that the vertical texture would increase the illusion relative to the no-texture no-frame condition of Experiment 1, and that the horizontal texture would decrease the illusion relative to that control. The grid texture would, of course, be expected to produce a magnitude of illusion intermediate between those of these two texture conditions.
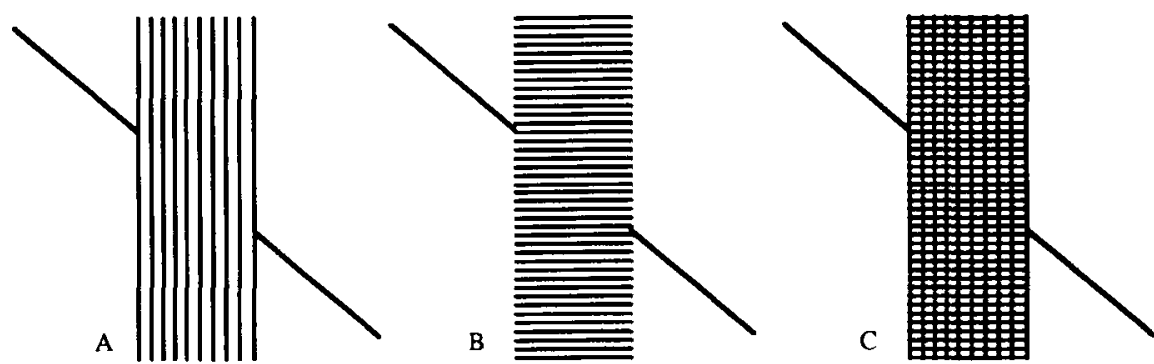

Figure 4. Examples of stimuli for Experiment 3. (A) Vertical texture. (B) Horizontal texture. (C) Grid texture. In the actual experimental stimuli, spatial frequency was constant throughout the texture conditions. 
However, the "narrowing of space" explanation of the Poggendorff illusion (e.g., Greist-Bousquet \& Schiffman, 1981) can also predict the same effects, but without appealing to a reference-frame component. If the vertical texture exacerbates the perceived narrowing of the space between the parallels, the illusion would increase. Likewise, the horizontal texture may inhibit the perceived narrowing of that space and thus decrease the illusion (although it is unclear, then, what is originally causing the perceived narrowing of space, because the horizontal texture has no vertical parallels at all). Therefore, although in Experiment 3 we tested the further susceptibility of the Poggendorff illusion to different types of spatial context, this did not permit an unambiguous discrimination between competing theories of the underlying mechanisms.

\section{Method}

Subjects. Eight undergraduates participated in this experiment to fulfill a course requirement. All had normal or corrected vision and were naive with regard to the experimental manipulations.

Apparatus. The design and apparatus were identical to those of Experiment 1, except that the trial sequence of all possible conditions was randomized. The breaks between every 20 trials, however, remained. In place of the frame and circle variables of Experiments 1 and 2 , we imposed a single three-level variable of texture orientation. (See Figure 4 for example stimuli.) There were 40 lines in the horizontal texture and 10 lines in the vertical texture. Both textures exhibited the same spatial frequency of 4 cycles/degree.

Procedure. Data collection proceeded in a fashion identical to that of Experiment 1.

\section{Results}

A repeated measures analysis of variance computed for the errors revealed two robust main effects of texture orientation and starting position. The horizontal texture condition (collapsed across rotations) produced the smallest illusory effect $(2.00 \mathrm{~mm})$, the vertical texture condition (collapsed across rotations) produced the greatest illusory effect $(3.00 \mathrm{~mm})$, and the grid texture condition (collapsed across rotations) produced an intermediate illusory effect $[2.56 \mathrm{~mm} ; F(2,14)=18.42, p<.001]$. (See Table 1.) Fisher's least significant difference tests were computed for cell comparisons of horizontal versus vertical texture $[t(7)=6.05, p<.001]$; of horizontal versus grid texture $[t(7)=3.41, p<.05]$; and of vertical versus grid texture $[t(7)=2.64, p<.05]$.

The mean error for trials in which the mobile transversal began in the lower starting position was $2.12 \mathrm{~mm}$,

Table 1

Mean Misalignments (in Millimeters) in Three Texture Conditions of Experiment 3 on Upright and Rotated Poggendorff Figures in Comparison With the Control Conditions (No Frame/No Texture) from Experiment 1

\begin{tabular}{|c|c|c|c|c|}
\hline & \multirow{2}{*}{$\begin{array}{l}\text { No Frame/ } \\
\text { No Texture }\end{array}$} & \multicolumn{3}{|c|}{ Texture } \\
\hline & & Vertical & Grid & Horizontal \\
\hline Upright figure & 2.93 & 3.27 & 2.58 & 2.13 \\
\hline Rotated figure & 1.87 & 2.73 & 2.54 & 1.86 \\
\hline Overall mean & 2.40 & 3.00 & 2.56 & 2.00 \\
\hline
\end{tabular}

whereas the mean error for trials with upper starting positions was $2.92 \mathrm{~mm}[F(1,7)=34.04, p<.002]$.

\section{Discussion}

The predicted differences between texture orientation conditions were borne out with surprising equality in distribution. The horizontal texture produced the smallest magnitude of illusion, the vertical texture the greatest magnitude of illusion, and the grid combination texture a magnitude of illusion almost perfectly in between those two. In fact, the magnitude of the illusion for the grid texture figure was very close to that of the no-texture noframe condition of Experiment 1 (averaged across rotations, $2.56 \mathrm{~mm}$ and $2.4 \mathrm{~mm}$, respectively). This is compelling evidence for equal and opposite influences of the additional horizontal and vertical inducing lines on the collinearity indexing process in the Poggendorff illusion. As stated before, it is likely that the Poggendorff illusion has several determinants (Day \& Kasperczyck, 1985; Weintraub et al., 1980). If only a portion of them were dependent on spatial context, it would make sense for the horizontal texture to merely reduce the illusion, rather than eliminate or reverse it.

This result contradicts Tong and Weintraub's (1974) finding that when internal "free-standing" vertical parallels are added to the Poggendorff display, the illusion decreases. Instead, this result allows a simpler version of the "cognitive mistracking" theory to explain the data. With more vertical lines between the parallels, the imaginary extension of one transversal to another becomes deviated more toward horizontal each time it crosses one of the lines. As this extension finally intersects the rightmost parallel well above the true collinearity, that position is judged to be too low.

Still, the "narrowing of space" explanation can also predict these data. Moreover, the effect of the horizontal lines may be explained simply by the fact that they produce an inverted Poggendorff that will have a directionally opposite illusion. However, it is not within the scope of this experiment to discriminate between different explanations of the specific process responsible for the Poggendorff illusion. Experiment 3 demonstrates that introducing more parallel lines to the Poggendorff display increases the illusion, at least in the rotated figure, whereas replacing them with many lines perpendicular to that orientation decreases the illusion, at least in the upright figure. This result is consistent with the reference-frame proposal, in that a particular one-dimensional reference orientation should produce a contextual influence that is opposite that of an orthogonal one, with the combination of the two canceling each other out.

\section{GENERAL DISCUSSION}

In the most general terms, these experiments show that the Poggendorff illusion is susceptible to spatial context. More specifically, a surrounding reference frame that is oriented with the transversals improves collinearity adjustments, but a surrounding frame that is not oriented 
with the transversals degrades collinearity adjustments. Curiously, this frame effect is not inhibited by a circumscribing circle (Ebenholtz \& Utrie, 1982, 1983). Finally, introducing several horizontal lines in place of the parallels improves collinearity adjustments (at least in the upright figure), and introducing several vertical lines in between the parallels degrades collinearity adjustments (at least in the rotated figure).

Although it does not explain the directionality of the traditional Poggendorff illusion, a reference-frame component to the Poggendorff illusion easily predicts these modulatory effects of spatial context. It first must attribute a direction-based contextual influence to the original parallels, in that they (possibly via "cognitive mistracking') cause the collinearity indexing process to be skewed toward the higher transversal's appearing too high and/or the lower transversal's appearing too low. Then, the visual reference frame that is oriented the same as the transversals provides an axis upon which collinearity of the transversals may be indexed (meanwhile, head-, body-, and gravity-centered reference frames will interfere with the visual reference frame, thus weakening its influence). In contrast, when the head-, body-, and gravity-centered reference frames are oriented with the transversals, the visual reference frame that is oriented differently will interfere with their facilitatory influence.

Clearly, a reference-frame theory alone is inadequate. Not only is it unable to predict the directionality of the Poggendorff illusion, but also it does not completely determine the magnitude of the illusion. However, the results of this study require that a reference-frame component to the Poggendorff illusion be acknowledged and considered in the identification and explanation of the mechanisms that underlie this illusion. In addition to explaining the effects of the surrounding frames, this proposal's value is shown in how it accounts for the reduction in the illusion for rotated Poggendorffs (Figures 1B and 1C), and for the persistence of the illusion when the parallels are removed (Figure 1D). Because the head-, body-, and gravity-centered reference frames will be aligned with the transversals of the rotated Poggendorff (when the viewer is upright), there is a coincident main line axis from those reference frames upon which collinearity of the transversals may be more accurately indexed. The fact that there are components of the Poggendorff illusion that are independent of referenceframe influences (together with the likelihood that the tilted visual reference frame provided by the parallels interferes with the other reference frames) explains why the illusion in the rotated Poggendorff does not completely vanish. Moreover, when the parallels are removed (the "obliqueline illusion"), head-, body-, and gravity-centered reference frames are upright and thus provide no main line axis for accurate collinearity indexing. Here, again, direction of the effect must be predicted by an accompanying theory.

There are likely to be several determinants of this illusion, only a subset of which are affected by spatial context. The studies presented here emphasize the importance of acknowledging the role of reference frames (and one- dimensional reference orientations) in understanding the Poggendorff illusion. Future work will require more indepth analysis of the underlying mechanisms to determine which ones involve reference frames and which ones do not.

\section{REFERENCES}

Asch, S. E., \& WrTkIN, H. A. (1948). Studies in space orientation: I. Experiments on perception of the upright with displaced visual fields. Joumal of Experimental Psychology, 38, 325-337.

Beh, H. C., Wenderoth, P. M., Purcell, A. T. (1971). The angular function of a rod-and-frame illusion. Perception \& Psychophysics, 9. 353-355

Bux, M. (1902). Die sog. Poggendorff sche optische Täuschungen. Scan dinavian Archives of Physiology, 13, 193-228.

Carmer, S. G., \& Swanson, M. R. (1973). An evaluation of ten pairwise multiple comparison procedures by Monte Carlo methods. Joumal of the American Statistical Association, 68, 66-74.

COREN, S. (1970). Lateral inhibition and geometric illusions. Quanterty Journal of Experimental Psychology, 22, 274-278.

DAY, R. H. (1973). The oblique line illusion: The Poggendorff effoct without parallels. Quarterly Journal of Experimental Psychology, 25, 535-541.

DAY, R. H., \& Dickinson, R. G. (1976). The components of the Poggendorff illusion. British Joumal of Psychology, 67, 537-552.

DAY, R. H., \& KASPERCZYCK, R. T. (1985). Apparent displacement of lines and dots in a parallel-line figure: A clue to the basis of the Poggendorff effect. Perception \& Psychophysics, 38, 74-80.

EBENHOLTZ, S. M. (1977). Determinants of the rod and frame effect: The role of retinal size. Perception \& Psychophysics, 22, 531-538.

EBENholtz, S. M. (1985a). Absence of relational determination in the rodand-frame effect. Perception \& Psychophysics, 37, 303-306.

EBENHoltz, S. M. (1985b). Blur-modulated orientation perception in the rod-and-frame task. Perception \& Psychophysics, 37, 109-113.

EaEnholtz, S. M. (1985c). Depth separation fails to modulate the orientation-inhibition effect. Perception \& Psychophysics, 37, 533-535.

Ebenholtz, S. M., \& UTRIE, J. W., JR. (1982). Inhibition of the rodand-frame effect by circular contours. Perception \& Psychophysics, 32 , $199-200$.

Ebenholtz, S. M., \& Utrie, J. W., JR. (1983). Peripheral circular contours inhibit the visual orientation control system. Aviation, Space, \& Environmental Medicine, 54, 343-346.

FindLAY, J. M., \& HoTOPF, W. H. N. (1985). Eye movements and illusions of alignment. Perception, 14, 387-391.

Gillam, B. (1971). A depth processing theory of the Poggendorff illusion. Perception \& Psychophysics, 10, 211-216.

Gillam, B. (1980). Visual illusions. Scientific American, 242, 102-112. Goldstein, M. B., \& Weintraub, D. J. (1972). The parallel-less Poggendorff: Virtual contours put the illusion down but not out. Perception \& Psychophysics, 11, 353-355.

GreEN, R. T., \& HoYLE, E. M. (1964). The influence of spatial orientation on the Poggendorff illusion. Acta Psychologica, 22, 348-366.

GrEGoRY, R. L. (1966). Eye and brain. London: Weidenfeld \& Nicolson.

Greist-Bousquet, S., \& SChiffman, H. R. (1981). The Poggendorff illusion: An illusion of linear extent? Perception, 10, 155-164.

HeLmholtz, H. voN (1962). Helmholtz's treatise on physiological optics (J. P. C. Southall, Trans.) New York: Dover. (Original work published 1910)

Hotopf, W. H. N. (1981), Mistracking in alignment illusions. Joumal of Experimental Psychology: Human Perception \& Performance, 7, 1211-1246

Hotopf, W. H. N., \& Ollerearnshaw, C. (1972). The regression to right angles tendency and the Poggendorff illusion: I. British Joumal of Psychology, 63, 359-367

Hotopf, W. H. N., Ollerearnshaw, C., \& Brown, S. (1974). The regression to right angles tendency and the Poggendorff illusion: III. British Journal of Psychology, 65, 213-231.

IMAl, S. (1973). Experiments on Poggendorff illusion. Joumal of Social Sciences \& Humanities, 90, 1-35.

Krantz, D. H., \& Weintraub, D. J. (1973). Factors affecting perceived 
orientation of the Poggendorff transversal. Perception \& Psychophysics, 14, 511-517.

Predebon, J. (1983). Recognition processes and occurrence of the dot Poggendorff illusion. Perceptual \& Motor Skills, 56, 471-474.

Pressey, A. W. (1967). Field dependence and susceptibility to the Poggendorff illusion. Perceptual \& Motor Skills, 24, 309-310.

Pressey, A. W. (1971). An extension of assimilation theory to illusions of size, area, and direction. Perception \& Psychophysics, 9, 172-176.

Quina, K., \& Pollack, R. H. (1973). Attraction of parallels as a function of intercontour distance. Perceptual \& Motor Skills, 36, 934.

SPIVEY-KNowlton, M. (1992). The virtual axis: A computational model of direct and indirect tilt illusions. Paper presented at the McDonnell Summer Institute of Cognitive Neuroscience, Dartmouth Medical School.

Tong, L., \& WeIntraub, D. J. (1974). Contour displacements and tracking errors: Probing 'twixt Poggendorff parallels. Perception \& Psychophysics, $15,258-268$.

Weintraub, D. J., \& Krantz, D. H. (1971). The Poggendorff illusion: Amputations, rotations, and other perturbations. Perception \& Psychophysics, 10, 257-264.

Weintraub, D. J., Krantz, D. H., \& Olson, T. P. (1980). The Poggendorff illusion: Consider all the angles. Joumal of Experimental Psychology: Human Perception \& Performance, 6, 718-725.

WENDEROTH, P. (1974). The distinction between the rod-and-frame illusion and the rod-and-frame test. Perception, 3, 205-212.

Wenderoth, P., Beh, H.[C.], \& White, D. (1978). Alignment errors to both ends of acute- and obtuse-angle arms. Perception \& Psychophysics, 23, $475-482$.
Wenderoth, P., \& Johnstone, S. (1988). The differential effects of brief exposures and surrounding contours on direct and indirect tilt illusions. Perception, 17, 165-176.

WENDEROTH, P., \& WADE, N. J. (1981). An investigation of line and dot forms of the Muller-Lyer and Poggendorff illusions. Quarterly Joumal of Experimental Psychology, 33A, 77-85.

WENDERoth, P., \& VAN DER ZWAN, R. (1989). The effects of exposure duration and surrounding frames on direct and indirect tilt aftereffects and illusions. Perception \& Psychophysics, 46, 338-344.

Wenderoth, P., van der Zwan, R., \& Johnstone, S. (1989). Mechanisms of orientation illusions. In D. Vickers, \& P. Smith (Eds.), Human information processing: Measures, mechanisms and models (pp. 83106). Amsterdam: North-Holland.

WiLSON, A. E. (1983). Assessment of apparent length and angle explanations of the Poggendorff effect. Perceptual \& Motor Skills, 57, 539-546.

WITKIN, H. A., \& AsCH, S. E. (1948). Studies in space orientation: IV. Further experiments on perception of the upright with displaced visual fields. Joumal of Experimental Psychology, 38, 762-782.

Wundt, W. (1893). Grundzüge der physiologischen Psychologie. Leip zig: Engelmann.

ZanutTini, L. (1976). A new explanation for the Poggendorff illusion Perception \& Psychophysics, 20, 29-32.

(Manuscript received July 26, 1991;

revision accepted for publication October 20, 1992.) 